\title{
Advance Care Planning Prior to Death in Older Adults with Hip Fracture
}

\author{
Anna Kata ${ }^{7}$ (D), Irena Cenzer ${ }^{7}$, Rebecca L. Sudore ${ }^{1,2}$, Kenneth E. Covinsky ${ }^{1,2}$, and \\ Victoria L. Tang ${ }^{7,3}$
}

'Division of Geriatrics, Department of Medicine, University of California, San Francisco, CA, USA; ${ }^{2}$ Division of Geriatrics, San Francisco Veterans Affairs Medical Center, San Francisco, CA, USA; ${ }^{3}$ Division of Hospital Medicine, Department of Medicine, Veterans Affairs Medical Center, San Francisco, CA, USA.

BACKGROUND: Although hip fractures in older adults are associated with a high degree of mortality and disability, the use of advance care planning (ACP) in this population is unknown.

OBJECTIVE: To determine the prevalence of ACP and need for surrogate decision-making prior to death in older adults with hip fracture and to identify factors associated with ACP.

DESIGN: Retrospective cohort study using Health and Retirement Study (HRS) interviews linked to Medicare fee-for-service claims data.

PARTICIPANTS: Six hundred six decedent participants aged 65 or older who sustained a hip fracture during HRS enrollment and had a proxy participate in the exit HRS survey.

MAIN MEASURES: Survey responses by proxies were used to determine ACP, defined by either advance directive completion or surrogate designation, and to assess decision-making at the end of life. Multivariate logistic regression was used to analyze correlates of ACP.

KEY RESULTS: Prior to death, $54.9 \%$ of all participants had an advance directive and 68.9\% had designated a surrogate decision-maker; however, $24.5 \%$ had no ACP. Of the total cohort, 32.5\% required decisions to be made about treatment at the end of life and lacked capacity to make these decisions themselves. In this subset, $19.9 \%$ had no ACP. In all participants, ACP was less likely in non-white individuals (adjusted odds ratio (aOR) $0.14,95 \%$ CI 0.06-0.31), those with less than a high school education (aOR 0.58, 95\% CI 0.35-0.97), and those with a net worth below the median of the cohort (aOR 0.49, 95\% CI 0.26-0.72). No clinical factors were found to be associated with ACP completion prior to death.

CONCLUSIONS: A considerable number of older adults with hip fracture required surrogate decision-making at the end of life, of whom one fifth had no ACP prior to

Prior Presentation Earlier versions of these findings were presented at the American Geriatrics Society Annual Meeting on May 2, 2019, in Portland, OR.

Electronic supplementary material The online version of this article (https://doi.org/10.1007/s11606-020-05644-1) contains supplementary material, which is available to authorized users.

Received April 10, 2019

Revised November 18, 2019

Accepted January 3, 2020

Published online May 4, 2020 death. Clinicians providing care for these patients are uniquely poised to address ACP.

KEY WORDS: advance care planning; older adults; hip fracture; advance directive; surrogate decision-making.

J Gen Intern Med 35(7):1946-53

DOI: $10.1007 / \mathrm{s} 11606-020-05644-1$

(C) Society of General Internal Medicine 2020

\section{INTRODUCTION}

\section{Background}

Hip fractures in older adults are common, resulting in over 300,000 hospitalizations in 2014 , and are associated with a high degree of mortality and disability. ${ }^{1}$ Half of those that incur this injury are over the age of 85 , and a significant proportion has declining health and function even before the hip fracture occurs. ${ }^{2,3}$ These factors, coupled with the approximately 20-30\% 1-year mortality rate, demonstrate the need for advance care planning (ACP) in older adults who sustain a hip fracture. ${ }^{4}$ Through ACP, individuals share their values and preferences regarding future medical care. This is typically done via advance directives (ADs), which are written statements of a person's wishes, and surrogate decision-makers, who are appointed to make medical decisions in the event an individual is unable to do so themselves. Ultimately, the goal of ACP is to receive medical care consistent with one's preferences. ${ }^{5}$

The importance of ACP for an older adult who is preparing to undergo surgery or has received a cancer diagnosis has been generally recognized. ${ }^{6,7}$ However, it is unclear if hip fractures, despite their serious nature and association with declining health trajectories, convey a similar urgency for ACP. While there are many factors that can influence whether an individual has ACP, for older adults who die at any time after a hip fracture, a lack of ACP can potentially indicate that the hip fracture was not recognized as an indicator for clinicians to initiate necessary ACP discussions. Currently, the prevalence of ACP prior to death in individuals who have sustained a hip fracture is unknown. Additionally, we do not know how often these patients lose decision-making capacity 
near the end of life and therefore would distinctly benefit from making their wishes known in advance. Two prior studies that measured ACP in this population were retrospective, limited to a small number of participants from a single center, and only assessed ACP documented in the medical record during the initial hospitalization; thus, these studies were unable to comment on ACP occurring outside these confines. ${ }^{4,8}$ A clear understanding of ACP and the factors associated with its absence at the end of life can provide clinicians with important contextual information to inform the care of this often frail and elderly population.

The current study evaluated the prevalence of ACP prior to death from any cause in those who sustained a hip fracture using a nationally representative cohort of older adults. Additionally, it determined the prevalence of surrogate decisionmaking at the end of life and examined clinical and demographic factors associated with ACP.

\section{PATIENTS/METHOD}

\section{Data Sources and Study Population}

This study used survey data from the Health and Retirement Study (HRS) linked to Medicare claims data (Fig. 1). HRS is a longitudinal study that biennially surveys health and economic factors in a nationally representative cohort of persons over the age of 50. ${ }^{9}$ When participants in HRS die, exit interviews are conducted with a proxy (a family member or knowledgeable informant) within 24 months of death. We constrained our study to HRS participants aged 65 or older, who had a documented hip fracture while enrolled in HRS between 1992 and 2014, subsequently died, and had a proxy complete an exit interview between 2000 and 2014. This study was approved by the Institutional Review Board of the University of California, San Francisco.

As done in previous studies, hip fractures were identified by linked Medicare fee-for-service data. ${ }^{3}{ }^{10}$ An HRS participant was deemed to have a hip fracture if one of the following two conditions was met: (1) the participant was admitted to a hospital with an admitting diagnosis International Classification of Disease, Ninth Revision (ICD-9) code for hip fracture or (2) a surgeon's charge for operative hip fracture repair (Current Procedural Terminology (CPT) code 27230-27248) supported with either (a) a second surgeon's charge within 2 days or (b) a supporting ICD-9 procedure code for hip fracture. We did not include admissions that were considered late effects from a prior hip fracture. The specific ICD-9 codes are listed in the Supplementary Material. For participants with more than one hip fracture, we used the first hip fracture that satisfied the inclusion criteria. From the 25,146 HRS participants aged 65 or older, 19,006 (76\%) had linked Medicare fee-for-service data, of which 1124 individuals had a hip fracture during their enrollment in HRS. Since we used Medicare claims to identify the presence of comorbid conditions, we excluded 107 participants who did not have 1 year of continuous Medicare enrollment prior to the hip fracture. We also excluded 160 participants who did not have an HRS interview within 2 years of the hip fracture. We then identified 748 who died before the end of the study period in 2014. Of those, we excluded 102 who did not have an exit interview between 2000 and 2014 as these were the survey years that included ACP questions, and 40 participants whose exit interview did not contain complete information regarding ACP. Our resulting cohort therefore included 606 participants.

\section{Measures}

Advance Care Planning. The outcome of ACP was defined as either completion of an $\mathrm{AD}$ or designation of a surrogate decision-maker. To assess the presence of an $\mathrm{AD}$ and designated surrogate, proxies were asked during the HRS exit interview, "Did [First Name] provide written instructions about the treatment or care [he/she] wanted to receive during the final days of [his/her] life?" and "Did [First Name] (also) make any legal arrangements for a specific person or persons to make decisions about [his/her] care or medical treatment if [he/she] could not make those decisions [himself/herself]? This is sometimes called a Durable Power of Attorney for Health Care."

Need for Surrogate Decision-Making. To assess whether medical decisions were required at the end of life, proxies were asked, "Did any decisions have to be made about the care and treatment of [First Name] during the final days of [his/her] life?" Decision-making capacity of the participant was determined by asking "Was [First Name] able to participate in decisions about [his/her] medical care during the final days of [his/her] life?" Thus, the need for surrogate decisionmaking was defined as the presence of necessary decisions to be made regarding end-of-life care coupled with the loss of decision-making capacity in the participant.

Sociodemographic and Clinical Correlates of ACP. Functional status, stratified by the presence of dependency in any activity of daily living (ADL) including bathing, dressing, eating, transferring, or toileting, and sociodemographic factors were assessed from the most recent HRS participant survey completed prior to the hip fracture. Medicare claims data from the index admission for hip fracture as well as all claims in the year prior to admission were used to determine the presence of dementia, cardiovascular and cerebrovascular diseases, and malignancy (ICD-9 codes listed in the Supplemental Material) and also to calculate overall comorbidity burden, using the Charlson Comorbidity Scale score. ${ }^{11}$ The specific comorbid conditions were chosen a priori given their potential to signal a limited life expectancy to clinicians and based on prior work done on ACP using HRS data. ${ }^{12}$ Expectation of death was assessed by an HRS exit interview survey question that asked proxies, "Was the death expected at about the time it occurred, or was it unexpected?" 


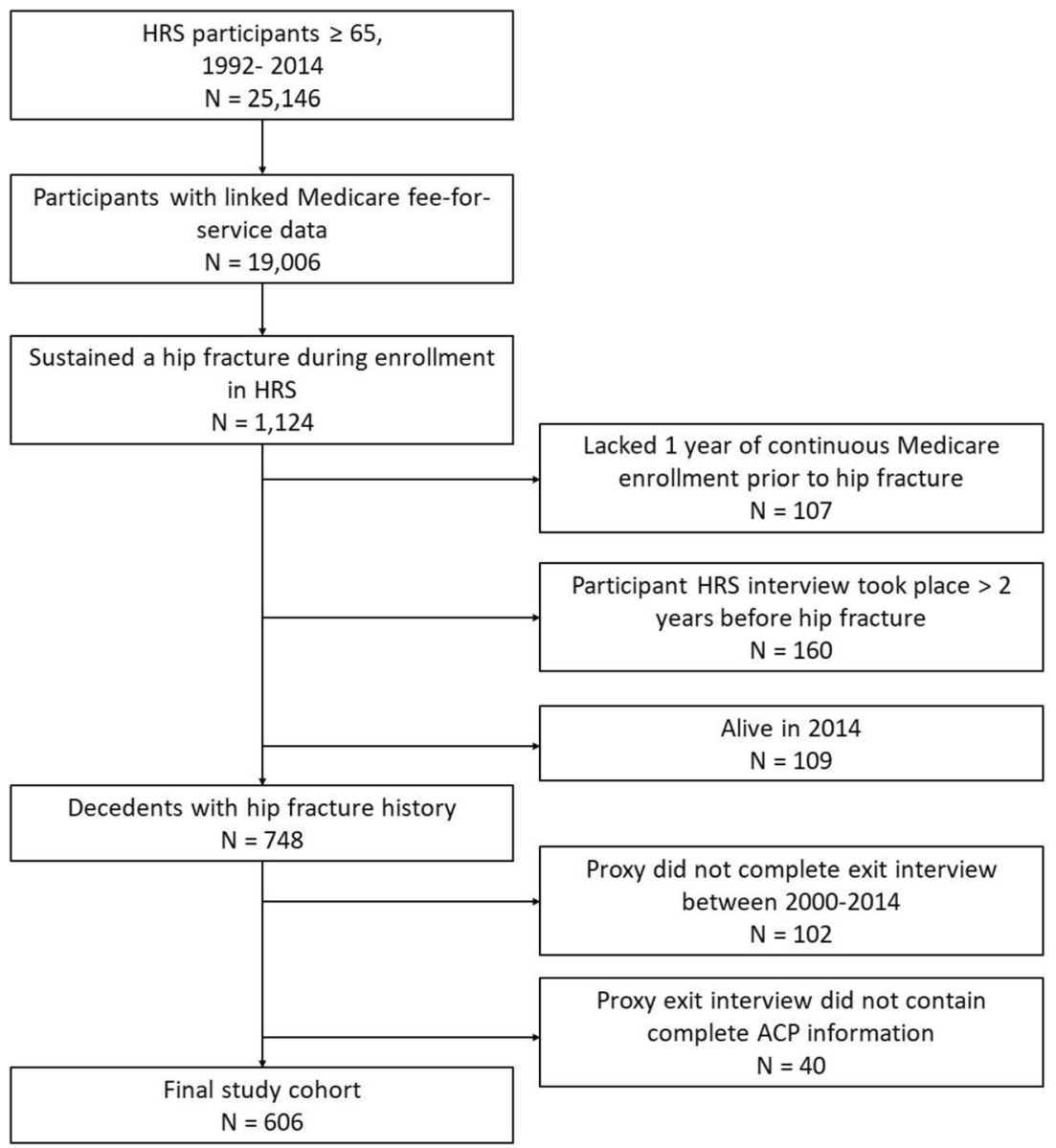

Fig. 1 Flowchart of creation of study cohort. HRS = Health and Retirement Study.

\section{Statistical Analysis}

We first tabulated the frequency of $\mathrm{AD}$ completion and surrogate designation for the entire sample and in the subset that required surrogate decision-making. We compared the characteristics of participants with and without ACP using chi-square tests for categorical variables and Mann-Whitney $U$ tests for continuous variables. Lastly, we used a multivariable logistic regression model to isolate the effect of each correlate on ACP completion. The variables were assessed for collinearity and were confirmed to have low variance inflation factors, indicating that collinearity was not present in the model. We took into account the complex design of HRS, and all analyses are weighted for participants' differential probability of selection into the HRS population. Statistical analyses were completed using Stata software (version 14; StataCorp, College Station, TX) and SAS (version 9.4; SAS Institute, Inc., Cary, NC).

\section{RESULTS}

\section{Characteristics of Study Population}

Of the total 606 HRS participants who sustained a hip fracture and subsequently died, the median age at the time of hip fracture was 84.4 years (IQR 79.2-88.9), $77.6 \%$ were female, $92.1 \%$ were white, and $10.5 \%$ resided in a nursing home (Table 1). Almost half had a high comorbidity burden (Charlson Comorbidity Scale score $>3,44.0 \%), 16.1 \%$ had a dementia diagnosis, and $27.8 \%$ had at least one ADL dependency. The average time between a participant's HRS survey and hip fracture was 12.5 months (standard deviation $(\mathrm{SD})=$ 7.8 months. The median time from hip fracture to death was 42.5 months (IQR 9.1-63.0), and for most participants $(66.4 \%)$, death was expected by the proxy when it occurred. The average time from participant death to proxy exit interview was 12.5 months ( $\mathrm{SD}=9.3$ months).

\section{Need for Surrogate Decision-Making at the End of Life}

Among the 606 participants, $41.6 \%(N=252)$ needed to make decisions regarding treatment at the end of life. Of the 252 participants who required decisions to be made, $21.8 \%(N=55)$ made these decisions themselves, while $78.2 \%(N=197)$ lacked decision-making capacity. Consequently, 197 participants of the total cohort of 606 $(32.5 \%)$ required surrogate decision-making at the end of life. 
Table 1 Baseline Characteristics of Older Adults with Hip Fracture by Advance Care Planning Prior to Death

\begin{tabular}{|c|c|c|c|c|}
\hline \multirow[t]{2}{*}{ Characteristic } & \multirow{2}{*}{$\begin{array}{l}\text { All participants }(N=606) \\
N(\%)\end{array}$} & \multirow{2}{*}{$\begin{array}{l}\text { No ACP }(N=168) \\
N(\%)\end{array}$} & \multirow{2}{*}{$\frac{\mathrm{ACP}(N=438)}{N(\%)}$} & \multirow[t]{2}{*}{$P$ value } \\
\hline & & & & \\
\hline Age at hip fracture (years), median (IQR) & $84.4(79.2-88.9)$ & $83.8(77.9-89.1)$ & $84.9(79.8-88.9)$ & 0.33 \\
\hline \multicolumn{5}{|l|}{ Age at hip fracture (years) } \\
\hline$<85$ & $289(49.6)$ & $84(53.0)$ & $205(48.6)$ & \multirow[t]{2}{*}{0.69} \\
\hline$\geq 85$ & $317(50.4)$ & $84(47.0)$ & $233(51.4)$ & \\
\hline Months between hip fracture and death, median (IQR) & $42.5(9.1-63.0)$ & $38.7(8.0-61.7)$ & $43.7(9.2-65.2)$ & 0.18 \\
\hline \multicolumn{5}{|l|}{ Gender } \\
\hline Female & 465 (77.6) & $113(66.5)$ & $352(81.3)$ & $<0.001$ \\
\hline \multicolumn{5}{|l|}{ Race } \\
\hline White & $532(92.1)$ & $117(78.0)$ & $415(96.7)$ & \multirow[t]{2}{*}{$<0.001$} \\
\hline Non-white & $74(7.8)$ & $51(21.94)$ & $23(3.3)$ & \\
\hline \multicolumn{5}{|l|}{ Marital status } \\
\hline Married or partnered & $193(30.2)$ & $60(35.3)$ & $133(28.5)$ & \multirow{2}{*}{0.12} \\
\hline Single* or widowed & $413(69.9)$ & $108(64.7)$ & $305(71.5)$ & \\
\hline Net worth, median (IQR), US\$ thousands & $77(6-250)$ & $30(0.6-125)$ & $106(12-275)$ & 0.002 \\
\hline \multicolumn{5}{|l|}{ Education } \\
\hline$\geq$ High school & $325(55.2)$ & $58(37.2)$ & $267(60.9)$ & \multirow[t]{2}{*}{$<0.001$} \\
\hline$<$ High school & $281(44.9)$ & $110(62.7)$ & $171(39.1)$ & \\
\hline \multicolumn{5}{|l|}{ Comorbidities $^{\dagger}$} \\
\hline Dementia & $94(16.1)$ & $32(20.7)$ & $62(14.6)$ & 0.09 \\
\hline Cardiovascular disease & $226(36.8)$ & $66(41.4)$ & $160(35.3)$ & 0.24 \\
\hline Cancer & $88(16.0)$ & $16(11.8)$ & $72(17.4)$ & 0.27 \\
\hline Cerebrovascular disease & $158(26.0)$ & $45(27.5)$ & $113(25.5)$ & 0.70 \\
\hline \multicolumn{5}{|l|}{ Charlson Comorbidity Scale score } \\
\hline$\leq 3$ & $345(56.0)$ & $96(54.2)$ & $249(56.6)$ & \multirow[t]{2}{*}{0.68} \\
\hline$>3$ & $261(44.0)$ & $72(45.8)$ & $189(43.4)$ & \\
\hline \multicolumn{5}{|l|}{ Function } \\
\hline 0 ADL dependency & $442(72.1)$ & $125(73.5)$ & $317(71.7)$ & \multirow{2}{*}{0.92} \\
\hline 1+ ADL dependency & $161(27.8)$ & $43(26.5)$ & $121(28.3)$ & \\
\hline Death expected ${ }^{\S}$ & $390(66.4)$ & $97(60.8)$ & $293(68.3)$ & 0.05 \\
\hline Nursing home resident" & $64(10.5)$ & $12(7.7)$ & $52(11.4)$ & 0.21 \\
\hline
\end{tabular}

Reported values incorporate survey weights to account the complex survey design of HRS

$A C P$ advance care planning, defined as having either an advance directive or designated surrogate decision maker; IQR interquartile range; ADL activity of daily living; HRS Health and Retirement Study

*Includes divorced and separated individuals

FIdentified with International Classification of Disease codes in Medicare claims linked to HRS data

†ADL dependency: needing assistance to compete an activity of daily living (bathing, dressing, eating, transferring, toileting), from HRS participant survey at time nearest to hip fracture

§Assessed by HRS exit interview survey question, "Was the death expected at about the time it occurred, or was it unexpected?"

IIFrom HRS participant survey at time nearest to hip fracture

Table 2 Advance Care Planning in Older Adults Who Did and Did Not Require Surrogate Decision-Making at the End of Life

\begin{tabular}{|c|c|c|c|c|}
\hline \multirow[t]{2}{*}{ Advance care planning } & \multirow{2}{*}{$\begin{array}{l}\text { All participants }(N= \\
\text { 606) } \\
N(\%)\end{array}$} & \multirow{2}{*}{$\begin{array}{l}\begin{array}{l}\text { Required surrogate decision- } \\
\text { making }(N=197)\end{array} \\
N(\%)\end{array}$} & \multirow{2}{*}{$\begin{array}{l}\text { Did not require surrogate decision- } \\
\text { making }(N=409) \\
N(\%)\end{array}$} & \multirow[t]{2}{*}{$\begin{array}{l}P \\
\text { value }\end{array}$} \\
\hline & & & & \\
\hline ACP* completed & $438(75.5)$ & $157(80.1)$ & $281(73.3)$ & 0.05 \\
\hline $\begin{array}{l}\text { Advance directive } \\
\text { completed }\end{array}$ & $315(54.9)$ & $117(59.6)$ & $198(52.7)$ & 0.09 \\
\hline \multicolumn{5}{|l|}{ Treatment preference ${ }^{\dagger}$} \\
\hline All care possible & $12(3.2)$ & $5(2.7)$ & $7(3.0)$ & 0.73 \\
\hline Limit care & $284(93.2)$ & $106(94.2)$ & $178(92.6)$ & 0.58 \\
\hline $\begin{array}{l}\text { Surrogate decision-maker } \\
\text { identified }\end{array}$ & $394(68.9)$ & $145(74.4)$ & $249(66.3)$ & 0.04 \\
\hline \multicolumn{5}{|l|}{ Surrogate preference } \\
\hline Spouse/partner & $62(16.3)$ & $19(13.5)$ & $43(17.8)$ & 0.48 \\
\hline Child/grandchild & $266(67.1)$ & $103(70.4)$ & $163(65.2)$ & \\
\hline Other & $63(16.6)$ & $23(16.1)$ & $40(16.9)$ & \\
\hline
\end{tabular}

Reported values incorporate survey weights to account the complex survey design of HRS

*Advance care planning (ACP), defined as having either an advance directive or designated surrogate decision maker

tTreatment preference specified in advance directive. All care possible and limit care were not mutually exclusive response options, \% calculated from completed advance directives

$\$$ Percentage calculated from identified surrogate decision-makers 
Table 3 Characteristics Associated with Advance Care Planning (ACP) Prior to Death in Older Adults with Hip Fracture

\begin{tabular}{|c|c|c|c|c|}
\hline \multirow[t]{2}{*}{ Characteristic } & \multicolumn{2}{|l|}{ Unadjusted } & \multicolumn{2}{|l|}{ Adjusted* } \\
\hline & OR $(95 \% \mathrm{CI})$ & $P$ value & OR $(95 \% \mathrm{CI})$ & $P$ value \\
\hline \multicolumn{5}{|l|}{ Age at hip fracture (years) } \\
\hline$<85$ & 1 [Reference] & - & 1 [Reference] & - \\
\hline$\geq 85$ & $1.19(0.83-1.7)$ & 0.69 & $1.11(0.63-1.96)$ & 0.71 \\
\hline \multicolumn{5}{|c|}{ Time between hip fracture and death (months) } \\
\hline$\geq 6$ & 1 [Reference] & - & 1 [Reference] & - \\
\hline$\overline{<} 6$ & $0.80(0.67-1.06)$ & 0.18 & $0.88(0.45-1.72)$ & 0.71 \\
\hline \multicolumn{5}{|l|}{ Gender } \\
\hline Male & 1 [Reference] & - & 1 [Reference] & - \\
\hline Female & $2.17(1.45-3.24)$ & $<0.001$ & $2.00(1.10-3.57)$ & 0.03 \\
\hline \multicolumn{5}{|l|}{ Race } \\
\hline White & 1 [Reference] & - & 1 [Reference] & - \\
\hline Non-white & $0.12(0.06-0.22)$ & $<0.001$ & $0.14(0.06-0.31)$ & $<0.001$ \\
\hline \multicolumn{5}{|l|}{ Marital status } \\
\hline Married or partnered & 1 [Reference] & - & 1 [Reference] & _ \\
\hline Single $^{\dagger}$ or widowed & $1.35(0.93-1.98)$ & 0.12 & $2.00(1.14-3.57)$ & 0.02 \\
\hline \multicolumn{5}{|l|}{ Net worth } \\
\hline$\geq$ Median of cohort & 1 [Reference] & - & 1 [Reference] & - \\
\hline$<$ Median of cohort & $0.43(0.28-0.58)$ & $<0.001$ & $0.49(0.26-0.72)$ & 0.002 \\
\hline \multicolumn{5}{|l|}{ Education } \\
\hline$\geq$ High school & 1 [Reference] & _- & 1 [Reference] & _- \\
\hline$<$ High school & $0.38(0.27-0.55)$ & $<0.001$ & $0.58(0.35-0.97)$ & 0.04 \\
\hline \multicolumn{5}{|l|}{ Comorbidities } \\
\hline Dementia & $0.65(0.41-1.03)$ & 0.09 & $0.64(0.35-1.15)$ & 0.13 \\
\hline Cardiovascular disease & $0.77(0.53-1.10)$ & 0.24 & $0.84(0.48-1.49)$ & 0.55 \\
\hline Cancer & $1.55(0.92-2.64)$ & 0.27 & $1.23(0.50-3.03)$ & 0.65 \\
\hline Cerebrovascular disease & $0.91(0.61-1.36)$ & 0.69 & $0.76(0.38-1.52)$ & 0.42 \\
\hline \multicolumn{5}{|c|}{ Charlson Comorbidity Scale score } \\
\hline$\leq 3$ & 1 [Reference] & - & 1 [Reference] & - \\
\hline & $0.92(0.63-1.29)$ & 0.68 & $1.20(0.62-2.32)$ & 0.57 \\
\hline \multicolumn{5}{|l|}{ Function $^{\ddagger 8}$} \\
\hline 0 ADL dependency & 1 [Reference] & - & 1 [Reference] & - \\
\hline 1+ ADL dependency & $1.08(0.72-1.61)$ & 0.92 & $1.12(0.64-2.00)$ & 0.66 \\
\hline Death expected" & $1.39(0.96-2.01)$ & 0.05 & $1.27(0.88-1.78)$ & 0.19 \\
\hline Nursing home resident ${ }^{\mathrm{q}}$ & $1.54(0.81-2.91)$ & 0.21 & $1.66(0.70-4.00)$ & 0.24 \\
\hline
\end{tabular}

Reported values incorporate survey weights to account the complex survey design of Health and Retirement Study (HRS). ACP is defined as having either an advance directive or designated surrogate decision maker

ADL activity of daily living

*Multivariate model adjusted for all characteristics listed in the table

Hincludes divorced and separated individuals

IIdentified with International Classification of Disease codes in Medicare claims linked to HRS data

\$ADL dependency: needing assistance to compete an activity of daily living (bathing, dressing, eating, transferring, toileting), from HRS participant survey at time nearest to hip fracture

"IAssessed by HRS exit interview survey question, "Was the death expected at about the time it occurred, or was it unexpected?" Reference group was death unexpected

IFrom HRS participant survey at time nearest to hip fracture

\section{Prevalence of Advance Care Planning}

At the time of their death, $54.9 \%$ of the 606 participants had completed an $\mathrm{AD}$ and $68.9 \%$ assigned a surrogate decisionmaker, while $24.5 \%$ had no ACP (Table 2 ). The vast majority of participants $(93.2 \%)$ who completed an $\mathrm{AD}$ expressed a preference for limiting care in certain situations, while $3.2 \%$ of those who completed an $\mathrm{AD}$ requested all care possible to prolong life. Among those who had a surrogate decisionmaker and were partnered, $32.1 \%$ chose their spouse or partner, while the remainder chose a child, grandchild, or another individual as their decision-maker.

In the subset of participants $(N=197)$ who required surrogate decision-making at the end of life, $59.6 \%$ completed an advance directive and $74.4 \%$ named a surrogate, while $19.9 \%$ had no ACP prior to death. The overall prevalence of ACP was similar in those that required surrogate decision making compared to those who did not require surrogate decision-making. However, participants who lacked the ability to make treatment decisions at the end of life were more likely to have a designated surrogate than those that retained the ability (74.4\% vs $66.3 \%$ ) (Table 2). There were no significant differences in care preferences expressed in ADs or surrogate identity between the two groups.

\section{Correlates of Advance Care Planning}

In a multivariate logistic regression analyzing the correlates of $\mathrm{ACP}$, defined as having either an $\mathrm{AD}$ or designated surrogate prior to death, participants were more likely to have ACP if they were female and single/widowed (Table 3). Participants were less likely have ACP if they were non-white, had less 
than a high school education, and had a net worth that was below the median of the cohort. Age, time between hip fracture and death, functional status, expectation of death occurring, nursing home residence, specific comorbidities, and overall comorbidity burden were not statistically significant correlates of ACP.

\section{DISCUSSION}

We found that half of older adults who sustained a hip fracture had an AD at the time of death, while just two thirds had a surrogate decision-maker. About one third lacked capacity to make required decisions at the end of life. Despite the need for surrogate decision-making in this subset, $19.9 \%$ had no form of ACP prior to death. For those who did make their treatment wishes known, most prioritized limiting treatment over receiving all care possible. In our multivariate logistic model, we showed that the likelihood of having ACP was lower for nonwhite individuals and those with lower levels of education and wealth. Clinical correlates such as age, comorbidities, and functional status had no association with ACP.

This study makes significant contributions to the current knowledge of ACP in individuals who sustain a hip fracture, with important implications for providers caring for these patients. By using a large nationally representative cohort, we were able to provide a more accurate estimate of ACP, determine what preferences were expressed, and analyze factors associated with ACP. In contrast, previous studies only measured ACP documented in the medical record, a source which has been shown to be often incomplete or inaccurate. ${ }^{4}$ 8,13 This can explain our finding of higher levels of ACP compared to a prior study, which reported that only $26.8 \%$ of patients admitted for a hip fracture had documented ACP. ${ }^{8} \mathrm{We}$ found rates of $\mathrm{ACP}$ comparable to other populations with serious illnesses. A study evaluating ACP prevalence in HRS participants who died from cancer found that $74 \%$ had surrogates and $50.7 \%$ had ADs, while another study showed that $41.7 \%$ HRS participants who died from end-stage renal disease had an $\mathrm{AD} .^{14,15}$

Among the 606 decedents, one third needed to make treatment decisions at the end of life but were unable to do so themselves, highlighting a group that would strongly benefit from ACP. Our results show that factors known to be associated with loss of decision-making capacity, such as dementia, increasing age, and nursing home status, are not associated with a higher likelihood of ACP. ${ }^{16,17}$ This means that a sizable proportion of those at highest risk of needing a surrogate decision-maker do not have one. Since ACP has been associated with improved quality of care at the end of life, causes of this gap should be addressed and greater effort should be placed by clinicians to engage in ACP discussions with patients in these subgroups. ${ }^{12}$ Importantly, we found that sociodemographic and socioeconomic factors were the primary correlates of ACP; non-white individuals and those with low levels of wealth and education were significantly less likely to have ACP. This finding aligns with prior work identifying racial and socioeconomic differences in ACP prevalence among older adults. ${ }^{18}$ Factors including health literacy, distrust of the medical system, spiritual beliefs, and reliance on family and community in decision-making have all been shown to impact ACP completion in minorities. ${ }^{19}$ Moreover, healthcare system issues, such as fragmentation of care or lack of a primary care physician, may make it more difficult to access already completed ACP documentation. ${ }^{20}$ Therefore, any interventions aimed at addressing ACP need to be implemented with these disparities in mind.

The median time to death in this population was just over 3 years from the hip fracture, highlighting a narrow window of opportunity for ACP discussions. The period directly following a hip fracture can be a time to start the process of ACP. However, engaging in ACP discussions while treating an acute injury can be challenging. One potential avenue of initiating the process of ACP in these patients is through co-management services, an increasingly popular model of care where a geriatrician or internist assists in the care of older adults hospitalized with a hip fracture. ${ }^{21}$ In addition to monitoring medical treatment, this clinician or another member of the team (e.g., social worker) can start to engage patients in ACP. Other strategies involve in-hospital providers communicating the need to initiate ACP discussions to a patient's primary care clinician. Research into strategies for engaging patients in ACP have shown that the most successful interventions relied on direct interactions between patients and healthcare professionals over multiple visits, making the outpatient primary care setting an ideal environment. ${ }^{22}$ Additionally, some have argued that the main objective of ACP should be to prepare patients and their surrogates for making inthe-moment medical decisions, rather than future treatment decisions. ${ }^{23}$ This requires surrogates to have a solid understanding of the patient's values as well as leeway in the process of decision-making. Clinicians can aid in this type of ACP by facilitating conversations between patients and their surrogates that focus on addressing these two issues. Ultimately, given the high mortality and morbidity associated with hip fractures, these injuries can be a harbinger of limited life expectancy. ${ }^{24}$ As such, we argue that all patients who have incurred a hip fracture are appropriate for ACP.

A few limitations of this study should be considered. First, information regarding ACP was provided by a proxy respondent, which allows for possible recall bias. However, despite this potential, prior studies have successfully used the HRS exit interviews to measure ACP at the end of life. ${ }^{12,16}$ Second, we only assessed the presence of a written advance directive or legal documentation of a surrogate, and so we were unable to capture more informal ACP such as discussions without subsequent documentation. Furthermore, the HRS survey only 
obtains detailed ACP information from the exit interview, which is obtained after the participant dies. Thus, we were unable to describe the prevalence of ACP in participants who had a hip fracture but had not died. However, given that we initially identified 857 HRS participants who sustained a hip fracture, this study captured the majority of the available population. Moreover, we were also unable to assess the care preferences of individuals who were lacking an advance directive or legally designated surrogate. Given that a common context for completing ACP documentation is in the setting of care limitation, it is not surprising that we found, like other studies, that most adults with ACP documentation state a preference for less aggressive care. ${ }^{12,16}$ In addition, despite looking at many possible correlates, we did not include geographic or regional characteristics, which may influence the prevalence of ACP. Lastly, as this study was not designed to determine the effects of ACP on end-of-life care in this cohort, we are unable to ascertain if ACP alters what treatments persons with hip fracture receive prior to death. However, prior research has shown that ADs do influence decisions made at the end of life. ${ }^{12,13}$

In conclusion, we found that a substantial proportion of older adults who sustain a hip fracture lack capacity to make necessary decisions at the end of life. However, even in this subset with a demonstrated need for surrogate decision-making, there remained a gap in ACP. Clinicians taking care of older adults who have a history of hip fracture are uniquely poised to address ACP. As such, strategies should be developed to incorporate this aspect of care into the long-term management of this population.

Corresponding Author: Anna Kata, Division of Geriatrics, Department of Medicine University of California, 4150 Clement Street, 306B, San Francisco, CA 94121, USA (e-mail: Anna.Kata@gunet. georgetown.edu).

Author Contributions The five listed authors were the sole contributors to this manuscript. AK, KC, VLT: study concept and design, and acquisition of the subjects and/or data. IC: data analysis. AK, IC, KC, RLS, VLT: interpretation of the results. AK: drafted the manuscript. All authors provided critical revision and approved the manuscript for publication.

Funding Information $A K$ was supported by the National Institute on Aging (NIA) at the National Institutes of Health (NIH) Research Training in Geriatric Medicine (T32 AG000212). VLT was supported in part by the NIA at the NIH (R03 AG056342). RS was supported in part by the NIA at the NIH (K24 AG054415). KC was supported in part by the NIA at the NIH (P3O AG044281, K24 AG029812). Funders had no role in the design and conduct of the study; collection, management, analysis, and interpretation of the data; and preparation, review, or approval of the manuscript.

\section{Compliance with Ethical Standards:}

Conflict of Interest: The authors declare that they do not have a conflict of interest.

\section{REFERENCES}

1. Health Care Utilization Project Network. Hospital Inpatient National Statistics 2014. https://hcupnet.ahrq.gov. Accessed October 9, 2018.

2. Brauer CA, Coca-Perraillon M, Cutler DM, Rosen AB. Incidence and Mortality of Hip Fractures in the United States. JAMA. 2009;302(14): 1573-1579.

3. Ritchie CS, Kelley AS, Cenzer IS, Smith AK, Wallhagen ML, Covinsky KE. High levels of geriatric palliative care needs in hip fracture patients before the hip fracture. J Pain Symptom Manag 2016;52(4):533-538.

4. Koso RE, Sheets C, Richardson WJ, Galanos AN. Hip Fracture in the Elderly Patients: A Sentinel Event. Am J Hosp Palliat Med 2018;35(4):612-619.

5. Sudore RL, Lum HD, You JJ, et al. Defining Advance Care Planning for Adults: A Consensus Definition from a Multidisciplinary Delphi Panel. J Pain Symptom Manag 2017;53(5):821-832.

6. Mohanty S, Rosenthal RA, Russell, MM, Neuman MD, Ko CY, Esnaola NF. Optimal Perioperative Management of the Geriatric Patient: A Best Practice Guideline from the American College of Surgeons NSQIP and the American Geriatrics Society. J Am Coll Surg 2016;222(5):930-47.

7. Ferrell BR, Temel JS, Temin S, Smith TJ. Integration of Palliative Care Into Standard Oncology Care: ASCO Clinical Practice Guideline Update Summary. J Oncol Pract 2017; 13(2): 119-121.

8. Dunn RH, Ahn J, Bernstein J. End-of-life Care Planning and Fragility Fractures of the Hip: Are We Missing a Valuable Opportunity? Clin Orthop Relat Res 2016;474(7):1736-1739.

9. Sonnega A, Faul JD, Ofstedal MB, Langa KM, Phillips JW, Weir DR. Cohort Profile: the Health and Retirement Study (HRS). Int $J$ Epidemiol 2014;43(2):576-585.

10. Fisher ES, Baron JA, Malenka DJ, et al. Hip fracture incidence and mortality in New England. Epidemiol Camb Mass 1991;2(2):116122.

11. Charlson ME, Pompei P, Ales KL, MacKenzie CR. A new method of classifying prognostic comorbidity in longitudinal studies: development and validation. J Chronic Dis 1987;40(5):373-383.

12. Bischoff KE, Sudore R, Miao Y, Boscardin WJ, Smith AK. Advance Care Planning and the Quality of End-of-Life Care among Older Adults. J Am Geriatr Soc 2013;61(2):209-214.

13. Walker E, McMahan R, Barnes D, Katen M, Lamas D, Sudore R. Advance Care Planning Documentation Practices and Accessibility in the Electronic Health Record: Implications for Patient Safety. J Pain Symptom Manag 2018;55(2):256-264.

14. Narang AK, Wright AA, Nicholas LH. Trends in advance care planning in cancer patients: Results from a national, longitudinal survey. JAMA Oncol 2015;1(5):601-608.

15. Wachterman MW, Lipsitz SR, Lorenz KA, Marcanonio ER, Li Z, Keating NL. End-of-Life Experience of Older Adults Dying of End-Stage Renal Disease: a Comparison w Cancer. J Pain Symptom Manag 2017;54(6): 789-97.

16. Silveira MJ, Kim SYH, Langa KM. Advance Directives and Outcomes of Surrogate Decision Making before Death. N Engl J Med 2010;362(13):1211-1218.

17. Raymont V, Bingley W, Buchanan A, et al. Prevalence of mental incapacity in medical inpatients and associated risk factors: crosssectional study. Lancet Lond Engl 2004;364(9443): 1421-1427.

18. Harrison KL, Adrion ER, Ritchie CS, Sudore RL, Smith AK. Low completion and disparities in advance care planning activities among older Medicare beneficiaries. JAMA Intern Med 2016;176(12):18721875.

19. Sanders JJ, Robinson MT, Block SD. Factors Impacting Advance Care Planning among African Americans: Results of a Systematic Integrated Review. J Palliat Med 2016;19(2): 202-27.

20. Mack JW, Paulk E, Visanath K, Prigerson HG. Black-White Disparities in the Effects of Communication on Medical Care Received Near Death. Arch Intern Med 2010;170(17): 1533-1540.

21. Kammerlander C, Roth T, Friedman SM, et al. Ortho-geriatric service-a literature review comparing different models. Osteoporos Int 2010;21(4):637-646. 
22. Ramsaroop SD, Reid MC, Adelman RD. Completing an advance directive in the primary care setting: What do we need for success? J Am Geriatr Soc 2007;55:277-283.

23. Sudore RL Fried, TR. Redefining the "Planning" in Advance Care Planning: Preparing for End-of-Life Decision Making. Ann Intern Med 2010; 153(4): 256-261.
24. Farahmand BY, Michaëlsson $\mathbf{K}$, Ahlbom A, Liunghall S, Baron JA; Swedish Hip Fracture Study Group. Survival after hip fracture. Osteoporos Int 2005; 16(12): 1583-90.

Publisher's Note Springer Nature remains neutral with regard to jurisdictional claims in published maps and institutional affiliations. 